\title{
Testing of Powders for Sensitivity to Air Effect Segregation
}

\author{
RJ Farnish ${ }^{1}$, P Kulkarni ${ }^{1}$, RJ Berry ${ }^{1}$, MSA Bradley ${ }^{1}$, V Guillebert ${ }^{2}$ \\ ${ }^{1}$ The Wolfson Centre for Bulk Solids Handling Technology, University of Greenwich, \\ Central Avenue, Chatham Maritime, Kent ME4 4TB, UK \\ ${ }^{2}$ Université de Rouen, Departement Mesures Physiques, \\ 76821 Mont Saint Aignan Cedex, France
}

\begin{abstract}
A loss of blend homogeneity through handling operations can have a major influence on the mechanical properties of sintered products. Plant optimisation to minimise the potential for segregation of blends can be undertaken through an audit of handling operations combined with an evaluation of the materials that are handled through the process. The correct identification of the mechanism of segregation is essential to support strategies to implement the most efficient and cost-effective counter measures. In support of this type of industrial activity, a piece of test apparatus has recently been developed to provide measurements of segregation potential for powders (metal and mineral) that are prone to loss of homogeneity (by composition or particle size) when subjected to counter-directional air displacements through equipment. This paper will discuss the test equipment and give examples of its output with respect to industrial application.
\end{abstract}

\section{$\underline{\text { Introduction }}$}

Handling processes commonly used in many powder metallurgy based plants often induce significant changes in either the feedstock or final blends. The causes of these changes can usually be attributed to equipment designs that deliver powders into the process such that either surface effect or air effect segregation occurs. The loss of homogeneity induced during filling operations is often exaggerated by equipment designs that are incapable of drawing evenly from the cross-sectional area of the inventory ${ }^{[1]}$. Usually useful improvements in blend stability can be obtained from the careful re-design of equipment such that the two main mechanisms of segregation are minimised.

The two main types of segregation can be found in many process plants, these are surface effect segregation and air effect segregation. It is not unusual to find that these mechanisms co-exist in the same process plant - with one or the other often being the more dominant at different handling stages.

The prediction of surface effect segregation (i.e. transportation of different particle size fractions during heap formation) has been the subject of a significant level of study ${ }^{[2,3,4,5]}$, and several techniques have been developed to simulate this mechanism of separation. Studies of air effect segregation have tended (until recently) to be limited to techniques whereby a bed of powder would be brought to a air fluidised condition followed by being kept in an expanded bed state for a controlled period of time. This approach effectively purging the bed to give a very strong segregation effect. Experimentation using this test technique with a range of powders demonstrated that dense and free-flowing powders were well suited to this approach, however it was also found that non free-flowing materials could not be adequately tested (their poor air permeability serving to prevent bed expansion and fluidisation). In order to overcome this limitation of test material types an improved tester was developed by The Wolfson Centre for Bulk Solids Handling Technology, University of Greenwich, UK ${ }^{[6,7]}$. 
Throughout this paper, graphs are presented to illustrate the range of segregation measured within test equipment. Irrespective of the type of tester used (surface or air effect), the starting point for the tests is clearly to determine the "virgin" size distribution for the samples under scrutiny. Test samples supplied for testing were typically 3 litres in volume. This material would be statistically divided using a spinning riffler to produce sets of sub samples. For some studies the basic test work would be augmented with SEM imaging and measurements of bulk density/particle density. Samples would typically be subjected to segregation testing and sub-samples would be constrained within the test apparatus for retrieval for analysis. The analysis would consider a comparison of the particle size distribution for two samples from extreme points within the tester. Data derived in this way would be processed into a graphical presentation whereby the size distribution from one point was subtracted from the size distribution at the other extreme of the tester. In this way values indicated above the median (zero) axis relate to an excess of an indicated size fraction in the upper sample, whilst values indicated below the median axis relate to an excess of an indicated size fraction in the lower sample. A value neither above nor below the median axis indicates that there is no difference between the extremes of samples obtained, and thus a homogenous state exists at that size fraction. An example of this type of graphical presentation is shown in Fig 1

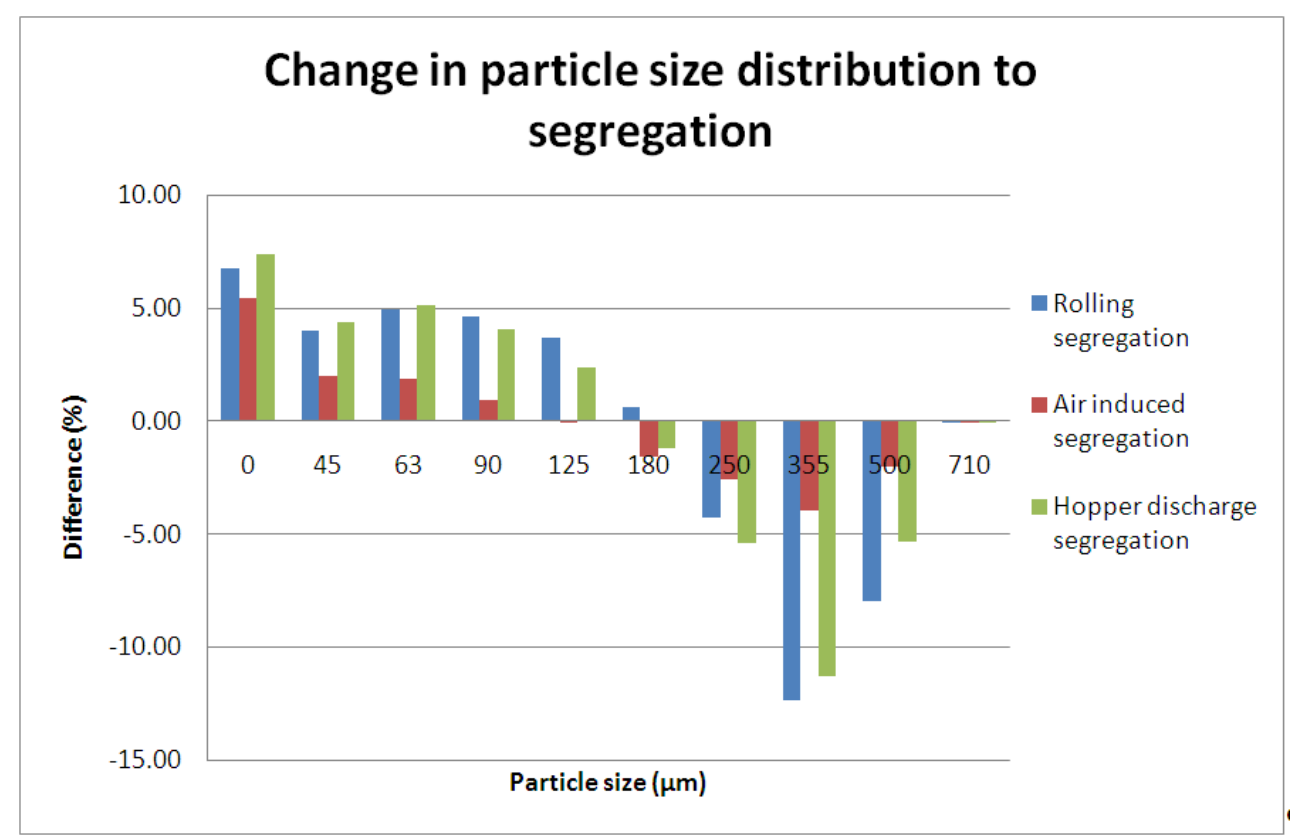

Fig 1 Graphical presentation of segregation characteristics measured in the laboratory compared to data obtained from a process

Fig 1 illustrates this presentation technique, but also serves to illustrate the point that materials can be sensitive to different mechanisms of segregation. In this particular example it is evident that the dominant mechanism of de-blending is that of surface effect segregation and that air effects are relatively minor in contributing to the process variability.

\section{Test Rig}

The dynamic air effect tester features a mass flow ${ }^{[4]}$ feed hopper which is interfaced onto a $50 \mathrm{~mm}$ bore quick acting slide valve. The test section beneath this point is constructed from stainless steel tube having a $52 \mathrm{~mm}$ bore. The test section is $450 \mathrm{~mm}$ tall and incorporates six $50 \mathrm{~mm}$ tall sub sample sections (two of which can be substituted with four $25 \mathrm{~mm}$ tall sections if required, for greater sample resolution). The lowermost element of the test section houses an air permeable membrane through which controlled supplementary counter directional air flows can pass. Fig 2 shows the layout of the test equipment. 


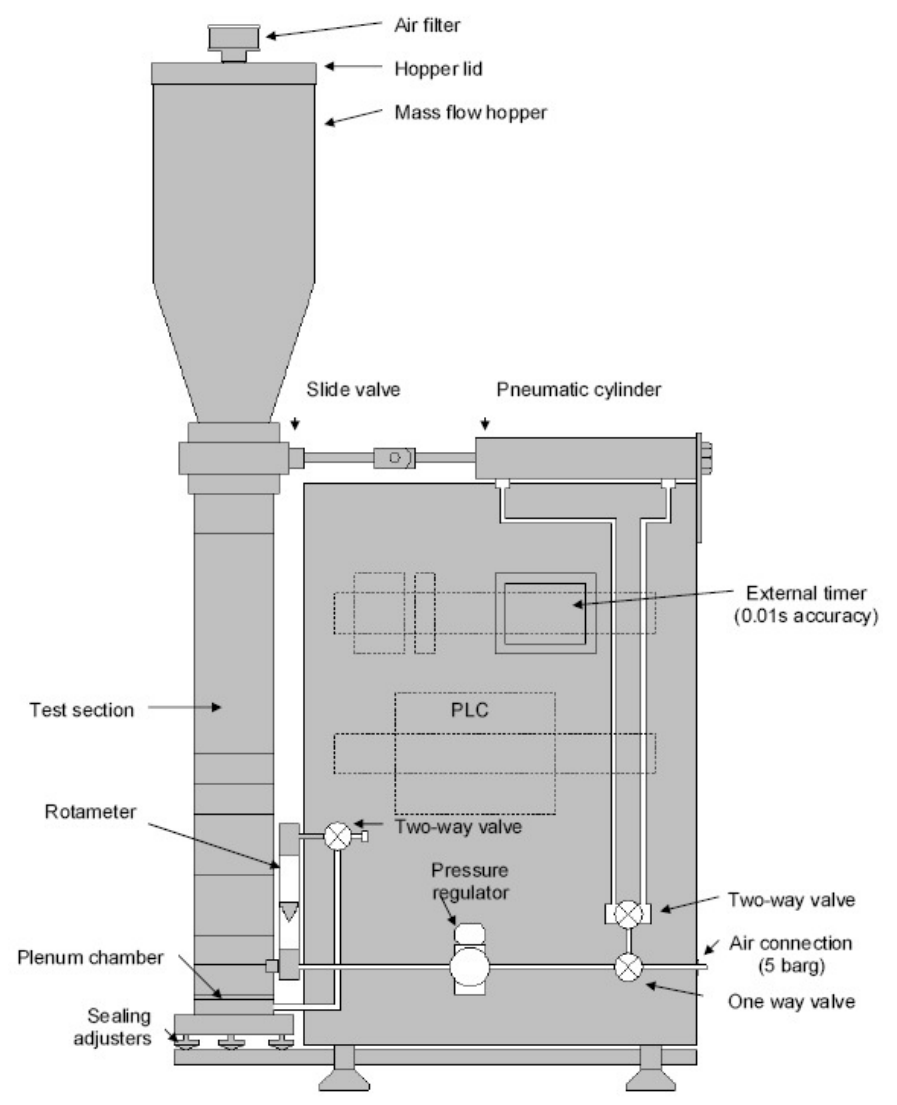

Fig 2 Illustration of the air effect segregation tester

The method of operation begins with the measuring out of a sample volume commensurate with the number of test sections in use (typically $\sim 200 \mathrm{ml}$ ). The sample is placed into the tester such that any segregation effects during loading are minimised (i.e. avoiding single point or off-centre loading). The test cycle commences with the activation of two separate timing devices. One timer operates the withdrawal of the slide gate under the hopper and the other initiates an introduction of gas through the base of the test section. The two timers are synchronised such that gas enters the test column 0.5 seconds before the slide gate opens, and continues to introduce gas for a further two seconds. After the gas introduction has ceased, the slide gate remains open for ten seconds to allow any entrained dust to settle back down into the test section. A PLC control system is installed to permit variations of the timing for the gas introduction (relative to the opening of the slide gate) and the duration of a counter-directional gas flow through the lower plenum section. These control parameters are in addition to a calibrated air flow meter external to the control panel (fed via a manually set pressure regulator).

The accumulated sample is captured within the test section and sub samples obtained by withdrawing the $50 \mathrm{~mm}$ rings (having fixed the sample in place between thin steel plates above and below the respective ring). The samples are then either subjected to size / shape analysis or forwarded to the client (for chemical analysis).

A very important benefit of this apparatus is that its design is such that a complete range of powdered or granular bulk particulates can be evaluated. The use of a mass flow feed hopper to hold the test sample in combination with a quick acting full bore slide valve permits 
the discharge of cohesive powder samples into the test section in a dilated state (simulating conditions present in many handling systems). This facilitates an interaction between the incoming charge of powder and the displaced (and augmented) counter directional air flow. The testing of cohesive powders in the current ASTM, by comparison, requires manual agitation of the packed bed of powder in order to prevent an upward transportation of the sample in the form of an impermeable "plug".

\section{$\underline{\text { Test programme }}$}

The apparatus can be used to obtain a single point characterisation of powders (an approach commonly used for benchmarking segregation sensitivity for a range of powders) - in which case it has been found that a supplementary air addition of 40 litres/min provides a good test resolution for metal powders.

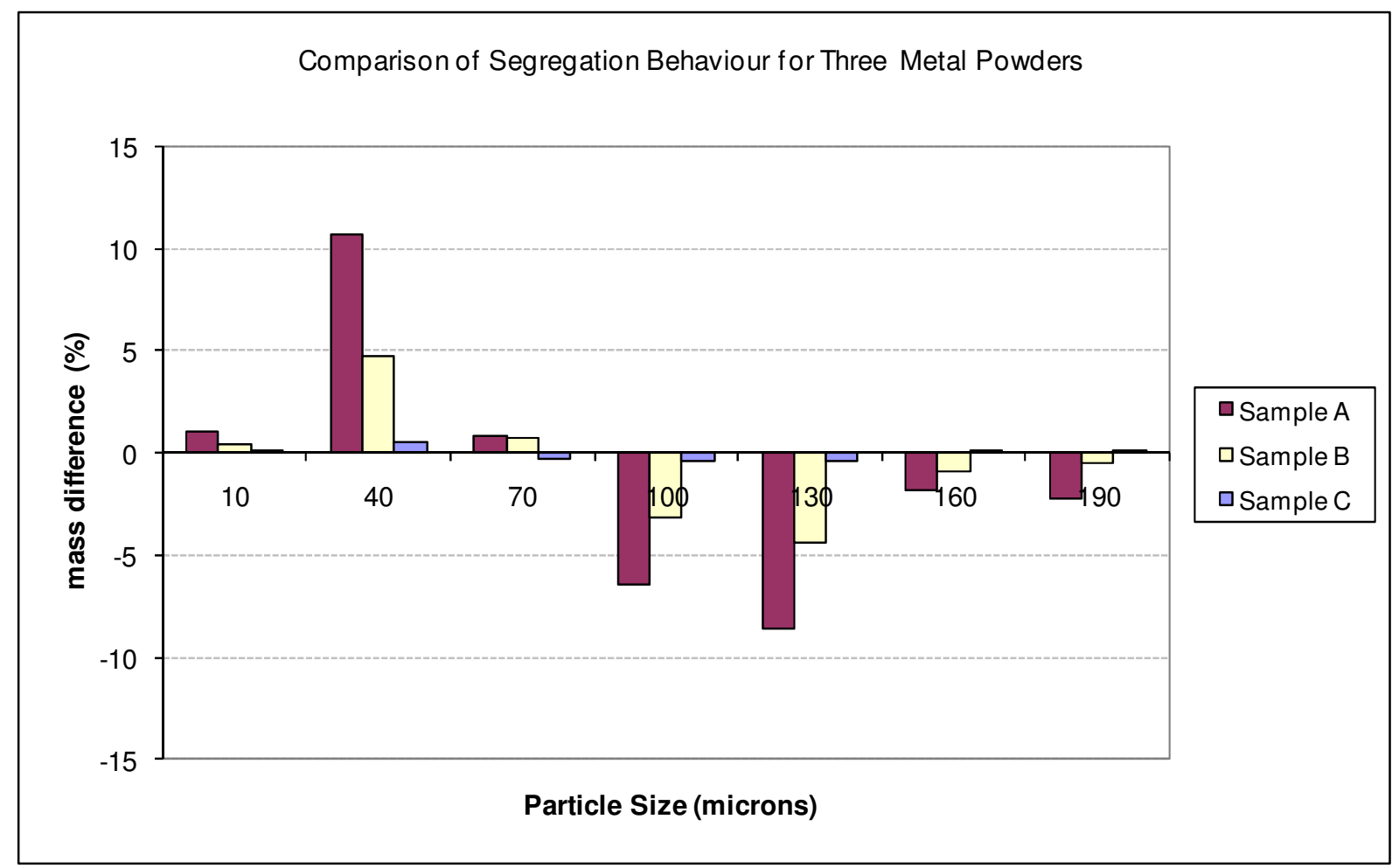

Fig 3 Comparison of segregation behaviour between three metal powders

Fig 3 shows how an evaluation of blend sensitivity can be presented such that a ranking of raw powders or blends can be arrived at. In this example it is clearly evident that metal sample $A$ is highly susceptible to segregation when subjected to counter directional air flows, whilst sample $\mathrm{C}$ represents a blend exhibiting a high level of blend stability under the same conditions. Referencing Fig 1, from earlier in this document, it should be borne in mind that air effect segregation is only one potential mechanism for de-blending and the relative insensitivity exhibit by sample $C$ to air effects may be driven by characteristics such as high particle density or high surface area - factors that may render this blend more sensitive to surface effect segregation!

For a standard analysis the data obtained from tests can be presented as per Fig 4 (size distribution from lowermost test sample subtracted from the uppermost), Fig 5 (size distributions from lowermost and uppermost shown on a common log graph) or Fig 6 (virgin size distribution subtracted from both of the size distributions obtained from the test rig). 


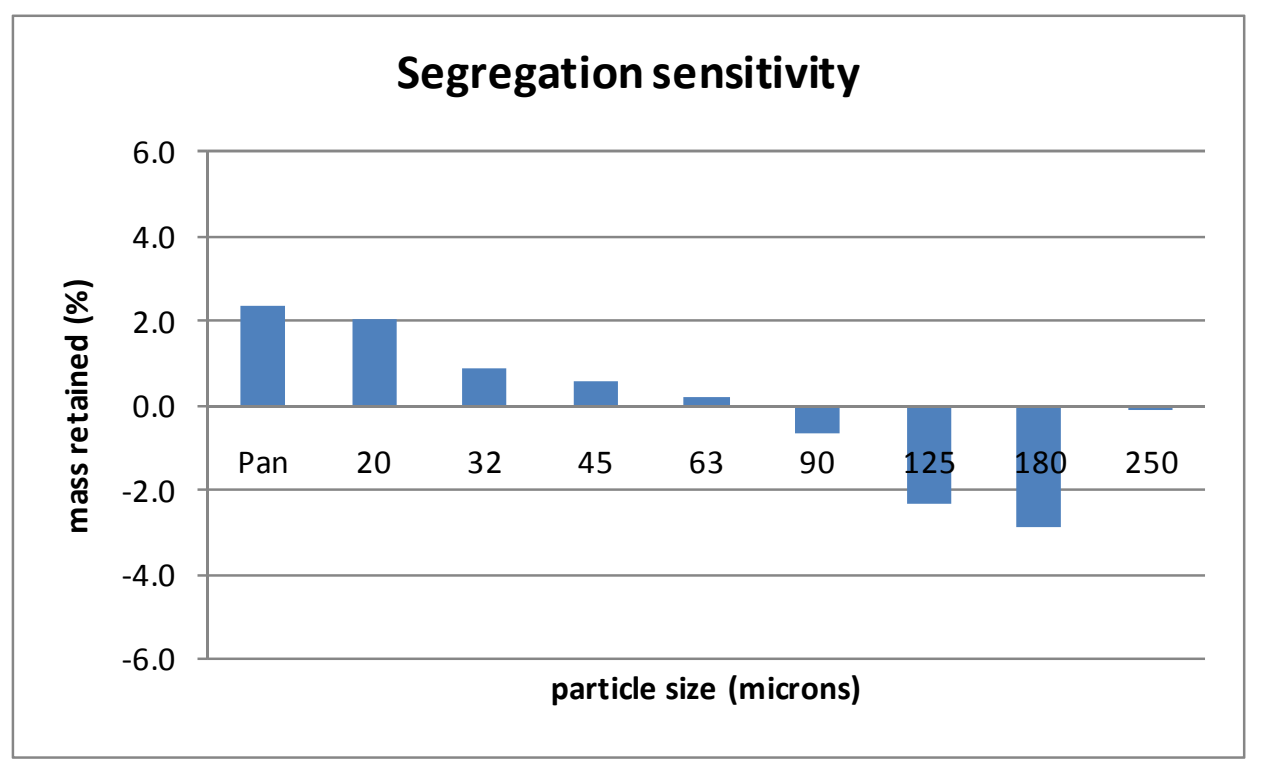

Fig 4 Presentation of test data comparing extremes of air effect segregation - graphical form 1

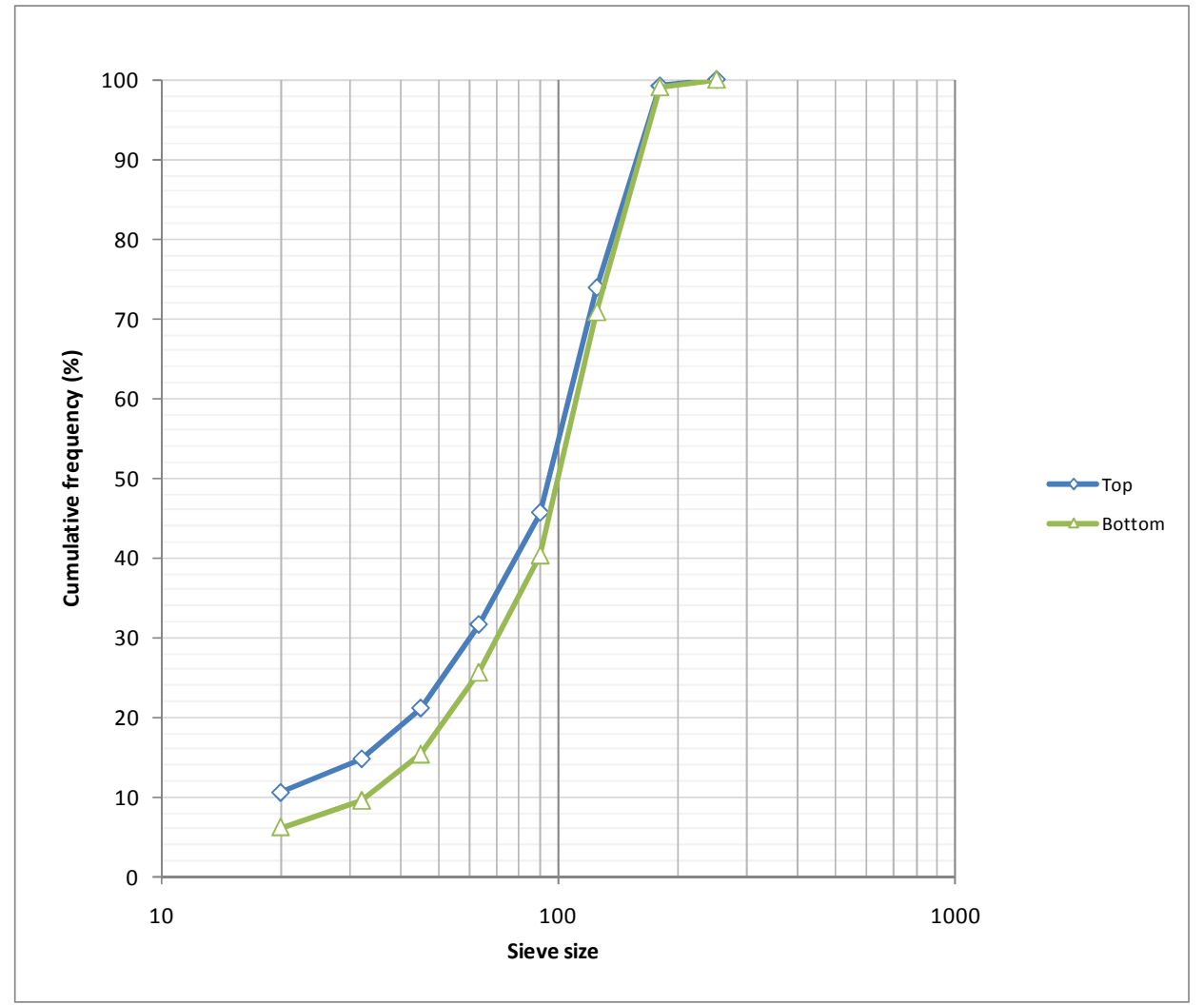

Fig 5 Presentation of test data comparing extremes of air effect segregation - graphical form 2 


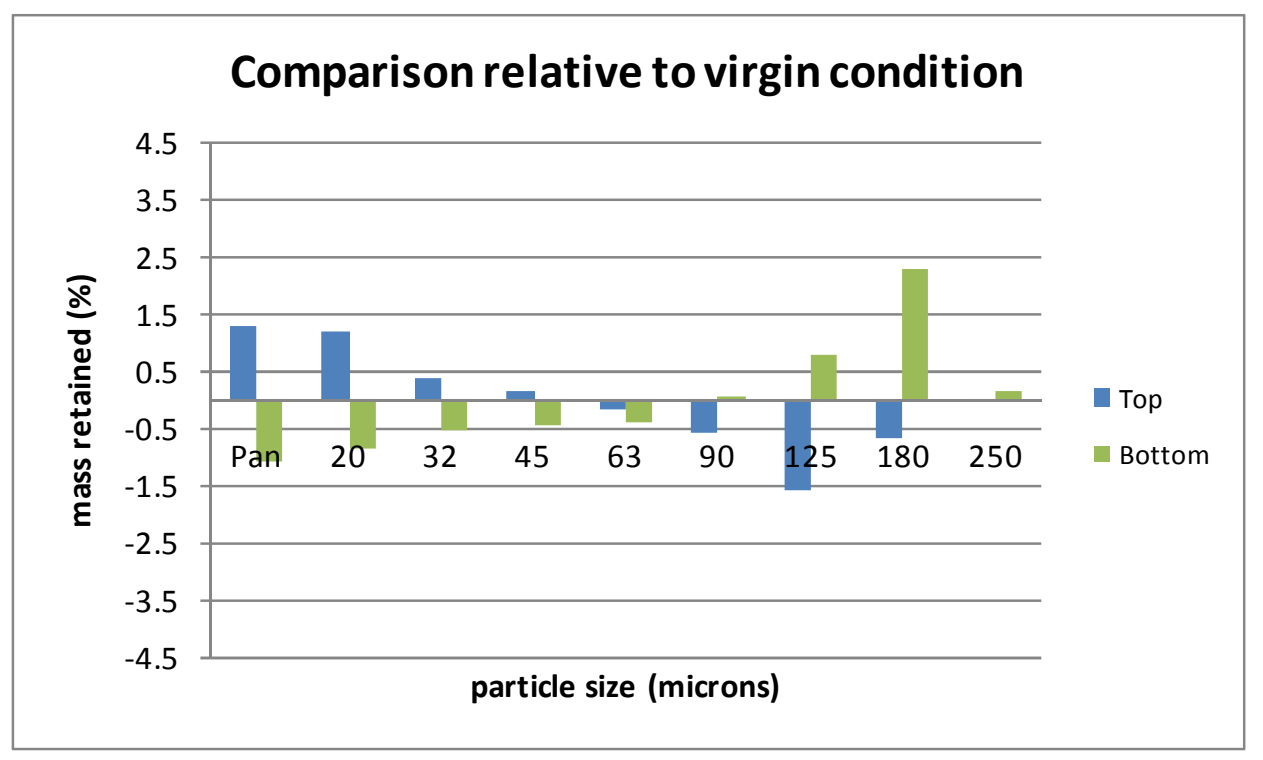

Fig 6 Presentation of test data from extremes of segregation relative to virgin condition - graphical form 3

The data obtained from an analysis of powders can be used to assess the blend stability of new blends prior to full scale manufacture, or it can be used to trouble shoot existing quality issues in process plants. Common counter-measures where a sensitivity to counter directional air flow exists, could include the re-design of equipment such that transfers of powder are achieved at conditions of high flux densities and low velocities. Dispersed deliveries of powder and excessive free-fall heights should be avoided.

\section{Conclusion}

The sensitivity of a wide range powders to air effect segregation can be evaluated using the automated segregation tester that has been developed by The Wolfson Centre for Bulk Solids Handling Technology, University of Greenwich. A significant advantage of the tester is that it can be used to characterise very fine powders and materials that may exhibit cohesion. The use of a feed vessel causes a dilation and flow of the test powder that replicates the dynamics of air effect segregation commonly found in many processes and equipment designs. The incorporation of adjustability of parameters such as, supplementary air flow rate, duration of air introduction and sequencing of powder release relative to air introduction endows the tester with a very useful degree of flexibility for operation as a conventional industrial tester or use as a research tool. 


\section{REFERENCES}

[1] Jenike \& Johanson Inc., Westford, MA, USA, US Patent 6487921

[2] Mosby, J., (1999), Investigations of the segregation of particulate solids with emphasis on the use of segregation testers, $\mathrm{PhD}$ thesis, Telemark College, Porsgrunn, Norway.

[3] Salter, G., (1999), Investigations into the segregation of heaps of particulate materials with particular reference to the effects of particle size, PhD Thesis, University of Greenwich, UK.

[4] Bridle I, Bradley M.S.A., Reed A.R., Abou-Chakra H., Tüzün U, Farnish R.J. Development of a test instrument to measure the segregation propensity of bulk materials, International Conference on Bulk Storage, Handling and Transportation 2004

[5] Dyrøy, A., (2006), Quantification and mitigation of segregation in the handling of alumina in aluminium production, PhD thesis, Telemark College, Porsgrunn, Norway.

[6] Farnish, R.J., P Kulkarni, RJ Berry, MSA Bradley, B Berthou, Segregation of bulk particulates subjected to counter directional air flows, Particulate Systems Analysis 2008, Stratford-upon-Avon, UK

[7] Farnish, RJ, Foucart, CN, Kulkarni, PA, Bradley, MSA, Reed, AR, Comparison of available techniques for characterising the air induced segregation tendencies of powders, International Conference on Bulk Storage, Handling and Transportation 2009 